\title{
Kolaviron Improved Resistance to Oxidative Stress and Inflammation in the Blood (Erythrocyte, Serum, and Plasma) of Streptozotocin-Induced Diabetic Rats
}

\author{
Omolola R. Ayepola, ${ }^{1}$ Nicole L. Brooks, ${ }^{2}$ and Oluwafemi O. Oguntibeju ${ }^{1}$ \\ ${ }^{1}$ Nutrition and Chronic Disease Research Unit, Oxidative Stress Research Centre, Department of Biomedical Sciences, \\ Faculty of Health and Wellness Sciences, Cape Peninsula University of Technology, Bellville, South Africa \\ ${ }^{2}$ Department of Wellness Sciences, Faculty of Health and Wellness Sciences, Cape Peninsula University of Technology, \\ Cape Town, South Africa
}

Correspondence should be addressed to Oluwafemi O. Oguntibeju; oguntibejuo@cput.ac.za

Received 4 January 2014; Accepted 17 February 2014; Published 24 March 2014

Academic Editors: C. M. Davis, E. Hopper-Borge, and C.-P. Wu

Copyright (C) 2014 Omolola R. Ayepola et al. This is an open access article distributed under the Creative Commons Attribution License, which permits unrestricted use, distribution, and reproduction in any medium, provided the original work is properly cited.

\begin{abstract}
Aims. Bitter kola seed (Garcinia kola, family: Guttiferae) has been used as a social masticatory agent in Africa for several years and is believed to possess many useful medicinal properties. The present study evaluates the antioxidative, anti-inflammatory, and antilipidemic effects of kolaviron (an extract from the Garcinia kola seeds) in the blood of streptozotocin- (STZ) induced diabetic rats. Methods. Diabetic rats were treated with kolaviron $(100 \mathrm{mg} / \mathrm{kg} \mathrm{b} \cdot \mathrm{wt})$ orally, five times a week for a period of six weeks. Serum glucose and $\mathrm{HBA}_{1 \mathrm{C}}$ concentrations were estimated in experimental groups. The activities of antioxidant enzymes: catalase (CAT), superoxide dismutase (SOD), and glutathione peroxidase (GPX) (in erythrocytes) as well as plasma concentration of malondialdehyde (MDA), a product of lipid peroxidation, oxygen radical absorbing capacity (ORAC) and ferric-reducing antioxidant power (FRAP) were investigated. Serum levels of proinflammatory cytokines and growth factor: interleukin- (IL) 1, monocyte chemotactic protein-1 (MCP-1), and vascular endothelial growth factor (VEGF), respectively, were also analyzed. Results. Kolaviron treatment markedly improved antioxidant status and abated inflammatory response evidenced by reduction in the levels of proinflammatory cytokines and growth factor, lipid peroxidation product, and the restoration of activities of erythrocyte antioxidant enzymes in the blood of diabetic rats. Conclusion. Kolaviron improved antioxidant status, reduced inflammation, and protected against hyperglycemic-induced oxidative damage in the blood of diabetic rats.
\end{abstract}

\section{Introduction}

Diabetes mellitus (DM) reduces life expectancy and adversely affects the quality of life of diabetic patients. According to the international diabetes federation (IDF), diabetic patients would increase from 371 million (2012) to 552 million in 2030. The limitations of the currently used antidiabetic drugs suggest an urgent need to discover new compounds that can serve as alternative and/or complementary therapy against this disease [1].

Hyperglycemia has been associated with an increased state of oxidative stress which is believed to play a crucial role in the onset and progression of late-diabetic complications through activation of stress-sensitive intracellular signaling pathways and the formation of gene products that causes cellular damage [2-4]. Biological free radicals are products of normal cellular metabolism and are maintained at a steady state level by antioxidants which act as free radical scavengers. At high concentrations, the production of free radicals overwhelms the detoxification capacity of cellular antioxidant system, resulting in oxidative stress and damage to cell structures $[5,6]$. Altered antioxidant enzyme activities have been reported in the blood of diabetic patients and in diabetic animal models. Red blood cells (RBCs) are the first cells in the body to be exposed to stressful stimuli and hence prone to oxidative stress [7]. Damage to red blood cells 
by reactive oxygen species (ROS) results in abnormalities in the function, morphology, and metabolism of erythrocyte $[8,9]$. Hyperglycemia and oxidation of membrane proteins are strongly associated with an increase in RBCs haemolysis and many pathological consequences [10]. Some of the mechanisms by which hyperglycemia causes oxidative stress include increased production of superoxide anion in the mitochondria [11], nonenzymatic glycation of proteins [12], and glucose autoxidation [13]. In addition, metabolic stress can result in changes in energy metabolism, reduced antioxidant defense, and increased levels of inflammatory mediators [14, 15].

Hyperlipidemia and altered antioxidant defenses are companions of oxidative stress. Diabetes-induced hyperlipidemia has been reported as one of the major risk factors for micro- and macrovascular complications [16]. Maintaining a balance between reactive oxygen species (ROS) and antioxidants is a major mechanism in preventing damage by oxidative stress; therefore, dietary supplementation of antioxidants could be a promising approach in the treatment of diabetes.

There is considerable interest in the potential beneficial effects of flavonoids on human health due to their biological activities which include antioxidant, antiviral, antiinflammatory, and antitumor activities [17]. Kolaviron (KV) is an extract from the bitter kola seeds (Garcinia kola, family: Guttiferae) containing a complex of Garcinia biflavonoids. $\mathrm{KV}$ has been proven to be beneficial in various pathological conditions of animal models through its antioxidative, antigenotoxic, analgesic, and anti-inflammatory properties [18-22], hence the need to explore its potentials in diabetic conditions. The present study investigated the beneficial effects of kolaviron on oxidative stress and inflammatory biomarkers in the blood of diabetic rats.

\section{Materials and Methods}

2.1. Chemicals. Streptozotocin (STZ), 6-hydroxydopamine, 6 -hydroxy-2,5,7,8-tetramethylchroman-2-carboxylic acid (trolox) and 2-thiobarbituric acid (TBA), and $\beta$-nicotinamide adenine dinucleotide phosphate reduced tetrasodium salt (NADPH) were obtained from Sigma-Aldrich (Johannesburg, South Africa). Malondialdehyde bis (diethyl acetal) (MDA), hexane, and methanol were purchased from Merck (Johannesburg, South Africa). All other chemicals and reagents used were of the highest commercially available purity.

2.2. Animals. The study protocol was approved by the Faculty of Health and Wellness Sciences Research Ethics Committee of the Cape Peninsula University of Technology (Ethics Certificate number CPUT/HW-REC 2012/AO4). All the animals received humane care in accordance with the criteria outlined in the "Guide for the Care and Use of Laboratory Animals" prepared by the National Academy of Science (NAS) and published by the National Institute of Health (Publication number 80-23, revised 1978). Male Wistar rats $(270 \pm 25 \mathrm{~g})$ were used for the study. Treatments were performed at the animal facility of the Medical Research Council (MRC),
South Africa, and all standard operating procedures (SOPs) were strictly adhered to. All animals were housed individually at room temperature $\left(22 \pm 2^{\circ} \mathrm{C}\right)$ with $55 \pm 5 \%$ humidity and an automatically controlled cycle of $12 \mathrm{~h}$ light and $12 \mathrm{~h}$ dark. Standard laboratory animal feed and water were provided ad libitum and animals were acclimatized to the experimental conditions for a period of one week before the commencement of the experiment.

2.3. Collection of Plant Material and Extract Preparation. Garcinia kola seeds were peeled, sliced, and air-dried (25$28^{\circ} \mathrm{C}$ ). Kolaviron was isolated according to the method of Iwu et al. [23]. Briefly, the powdered seeds were extracted with light petroleum ether (bp $40-60^{\circ} \mathrm{C}$ ) in a soxhlet for $24 \mathrm{hr}$. The defatted dried product was repacked and extracted with acetone. The extract was concentrated and diluted twice its volume with water and extracted with ethylacetate $(6 \times$ $300 \mathrm{~mL}$ ). The concentrated ethylacetate yielded kolaviron, a golden yellow solid.

2.4. Experimental Design. Diabetes was induced in overnight fasted rats by a single intraperitoneal injection of a freshly prepared solution of streptozotocin (STZ; $50 \mathrm{mg} \mathrm{kg}^{-1}$ body weight) in citrate buffer (0.1 M, pH 4.5). Five days after STZ injection, diabetes was confirmed by a stable hyperglycemia $(>18 \mathrm{mmol} / \mathrm{L})$ in the tail blood glucose with a glucometer (Accu-Chek, Roche, Germany). The animals were divided into 4 groups ( $n=10$ per group): normal control (NC group), kolaviron treated normal control (KV), diabetic control (DM group), and kolaviron-treated diabetic group (DM $+\mathrm{KV}$ group). Kolaviron was dissolved in vehicle (dimethylsulphoxide (DMSO)) and administered orally at a dose of $100 \mathrm{mg} \mathrm{kg}^{-1}$ five times a week for six weeks. Normal control (NC) rats also received vehicle throughout the study period.

For biochemical estimations in the blood, rats were sacrificed under sodium pentobarbital anesthesia $(60 \mathrm{mg} / \mathrm{kg})$. Random blood glucose was determined in rats after collection of blood specimen from the abdominal aorta into glucose tubes. Blood samples were collected into tubes with or without EDTA to obtain plasma or serum, respectively, and centrifuged at $3500 \mathrm{~g}$ for $10 \mathrm{~min}$ at $4^{\circ} \mathrm{C}$. Blood was also collected into another set of tubes used for $\mathrm{HBA}_{1 \mathrm{C}}$ estimation. Erythrocytes were obtained from EDTA-treated blood after plasma separation. Buffy-coat layers were discarded and erythrocytes were washed three times with cold saline and centrifuged at $3000 \mathrm{rpm}$ for $10 \mathrm{~min}$. Samples were haemolyzed by the addition of a threefold volume of icecold double distilled water $\left(\mathrm{ddH}_{2} \mathrm{O}\right)$ and the haemolysate was obtained after removing the cell debris by centrifugation at $3000 \mathrm{rpm}$ for another $10 \mathrm{~min}$. The supernatant was collected and stored at $-80^{\circ} \mathrm{C}$ prior to the estimation of enzymatic activity.

2.5. Analysis of Glucose, Glycated Haemoglobin, and Lipid Profile. Plasma glucose, glycated haemoglobin $\left(\mathrm{HBA}_{1 \mathrm{C}}\right)$, and serum lipid profile (total cholesterol, triglycerides) were analyzed with diagnostic kits in an automated clinical chemistry analyzer (Medical Cooperation, Bedford, MA, USA). 
2.6. Plasma Antioxidant Capacity Assays. The antioxidant capacity of plasma samples was determined by the ferricreducing antioxidant power (FRAP) assay of Benzie and Strain [24] with slight modifications in a Multiskan Spectrum plate reader (Thermo Fischer Scientific, Waltham, MA, USA). Oxygen radical absorbance capacity (ORAC) assay was conducted to kinetically measure the peroxyl radical scavenging activity in plasma samples with trolox as the antioxidant standard according to the method of Ou et al. [25]. The total plasma polyphenol was performed using the Folin Ciocalteu's phenol reagent according to the method of Singleton et al. [26].

2.7. Erythrocyte Antioxidant Enzyme Activity Assays. Activities of antioxidant enzymes in the erythrocytes were estimated in a clear 96-well plate using a Multiskan Spectrum plate reader (Thermo Fisher Scientific, USA). Catalase (CAT) activity was determined by the method of Aebi [27]. Superoxide dismutase was determined by the method of Crosti et al., [28], based on the inhibitory effect of SOD on the spontaneous autoxidation of 6-hydroxydopamine. Glutathione peroxidase (GPX) activity was determined according to the method of Ellerby and Bredesen [29] based on the oxidation of NADPH to NADP ${ }^{+}$in the presence of $\mathrm{H}_{2} \mathrm{O}_{2}$. The protein concentrations of the erythrocyte were determined by the bicinchoninic acid (BCA) kit (Pierce, Illinois, USA).

2.8. Lipid Peroxidation (LPO). Plasma malondialdehyde (MDA), an end product of lipid peroxidation, was determined by High-Performance Liquid Chromatography (HPLC) using a method adapted from Khoschsorur et al. [30]. Briefly, $100 \mu \mathrm{L}$ of plasma samples and standard MDA were mixed with $750 \mu \mathrm{L}$ orthophosphoric acid $(0.44 \mathrm{M})$, $250 \mu \mathrm{L}$ of aqueous thiobarbituric acid $(42 \mathrm{mM})$, and $450 \mu \mathrm{L}$ distilled water. The mixture was heated in a boiling water bath for $60 \mathrm{~min}$. After cooling on ice, alkaline methanol (50 mL methanol $+4.5 \mathrm{~mL} 1 \mathrm{M} \mathrm{NaOH})$ was added $(1: 1)$. The samples were centrifuged at $3500 \mathrm{~g}$ for $3 \mathrm{~min}$ at $4^{\circ} \mathrm{C} .1 \mathrm{~mL}$ of supernatant was added to $500 \mu \mathrm{L}$ of $\mathrm{n}$-hexane and the mixture centrifuged at $14000 \mathrm{~g}$ for $40 \mathrm{sec} .50 \mu \mathrm{L}$ of the supernatant was then chromatographed on an Agilent 1200 series HPLC. A $5 \mu \mathrm{m}$ YMC-PackPro $\mathrm{C} 18(150 \mathrm{~mm} \times 4.6 \mathrm{~mm}$ i.d. $)$ column was used for separation with $60: 40(\mathrm{v} / \mathrm{v}) 50 \mathrm{mM}$ phosphate buffer ( $\mathrm{pH}$ 6.8) and methanol, respectively, as mobile phase. The flow rate was $1 \mathrm{~mL} \mathrm{~min}^{-1}$. Fluorometric detection was performed with excitation at $532 \mathrm{~nm}$ and emission at $552 \mathrm{~nm}$. The peak of the MDA-TBA adduct was calibrated with the MDA standard.

2.9. Assay of IL-1, MCP-1, and VEGF. The serum levels of inflammatory markers including monocyte chemotactic protein-1 (MCP-1), vascular endothelial growth factor (VEGF), and interleukin (IL)-1 were measured in the serum using Bio-Plex Pro magnetic bead-based assays (Bio-Rad Laboratories, Hercules, USA) on the Bio-Plex platform (BioRad). Following previous optimization, samples were evaluated undiluted in a blinded manner. Samples were reacted with a mixture of fluorescent polystyrene beads bound with specific anticytokine primary antibodies, resulting in binding of the cytokines to the beads with the corresponding antibody. The biotinylated anticytokine secondary antibodies were then added and allowed to bind to the cytokine-bead complex followed by the addition of fluorescent phycoerythrin-conjugated streptavidin. All analytes levels in the quality control reagents of the kits were within the expected ranges. The standard curve for all the analytes ranged from 3 to $12000 \mathrm{pg} / \mathrm{mL}$. Bio-Plex Manager software, version 6.0, was used for bead acquisition and analysis.

2.10. Statistical Analysis. Values were expressed as mean \pm $\mathrm{SD}$. Data were tested for normality and equality of variance using the Levene's test. Differences between groups mean were estimated using one-way analysis of variance (ANOVA) followed by the Student-Newman-Keuls test for all pairwise comparisons. The Kruskal-Wallis test, a nonparametric analogue to the one-way ANOVA, was used to test for group differences when data was not normally distributed. Result were considered statistically significant at $P<0.05$ or marginally significant at $P<0.1$. All the statistics were performed using MedCalc version 12.2.1 software (MedCalc software bvba, Mariakerke, Belgium).

\section{Results}

3.1. Kolaviron Treatment Lowered Blood Glucose, Glycated Haemoglobin $\left(H B A_{1 C}\right)$, and Levels of Lipid Profiles. Kolaviron treatment reduced total cholesterol and triglyceride concentrations in the serum of normal and diabetic rats as shown in Figure 1. Serum glucose concentration of the diabetic group was 2.84-fold higher than the nondiabetic group, indicating a sustained hyperglycemic state in the STZinduced diabetic rats. Glycated hemoglobin $\left(\mathrm{HBA}_{1 \mathrm{C}}\right)$ was significantly elevated in diabetic rats and the administration of kolaviron significantly lowered blood glucose and $\mathrm{HBA}_{1 \mathrm{C}}$ levels in diabetic rats.

3.2. Kolaviron Alleviates Oxidative Stress in the Erythrocyte of Diabetic Rats. A significant increase in GPX and SOD activities was observed in the erythrocytes of diabetic rats compared to control group (Table 1). This alteration was reversed after kolaviron administration to STZ-induced diabetic rats for 6 weeks. No significant change in CAT activity was observed. Diabetic rats showed increased level of the lipid peroxidation product, MDA. Kolaviron significantly reduced the formation of plasma MDA in STZ-diabetic model.

3.3. Effect of Kolaviron Treatment on Plasma Antioxidant Capacity. Although an increasing trend was observed in plasma antioxidant status assessed as FRAP, ORAC, and total polyphenols in diabetic rats, following kolaviron treatment for 6 weeks (Table 2), no statistically significant difference was observed in the estimated parameters in all treatment groups.

3.4. Kolaviron Abates Inflammation in the Serum of Diabetic Rats. Diabetic rats had significantly elevated serum levels of MCP-1, VEGF, and IL-1 $\beta$ compared to control rats (Table 3) 


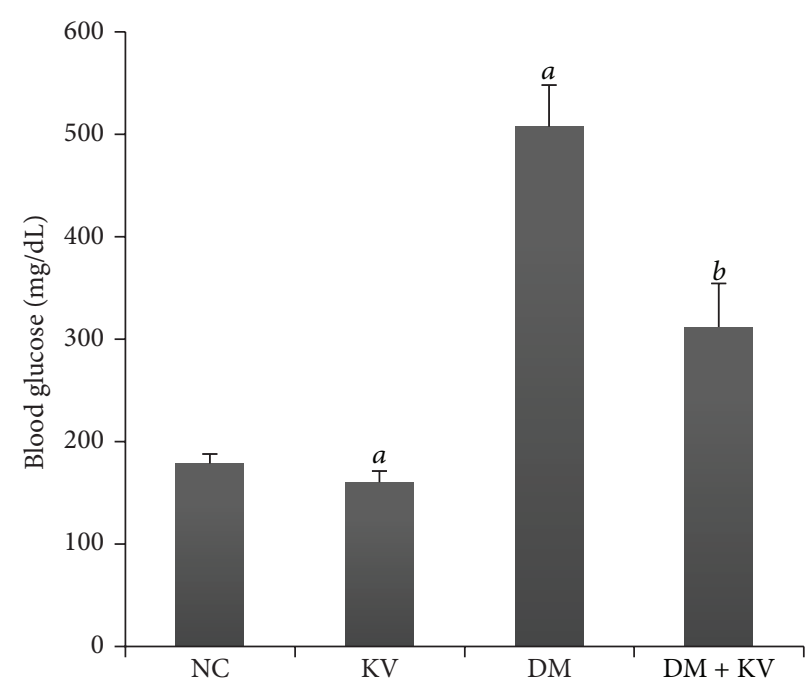

(a)

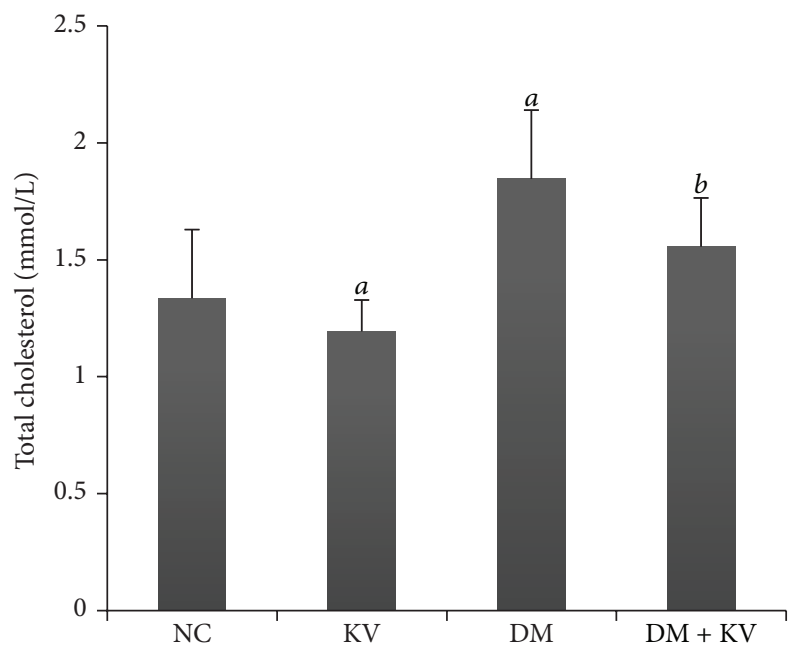

(c)

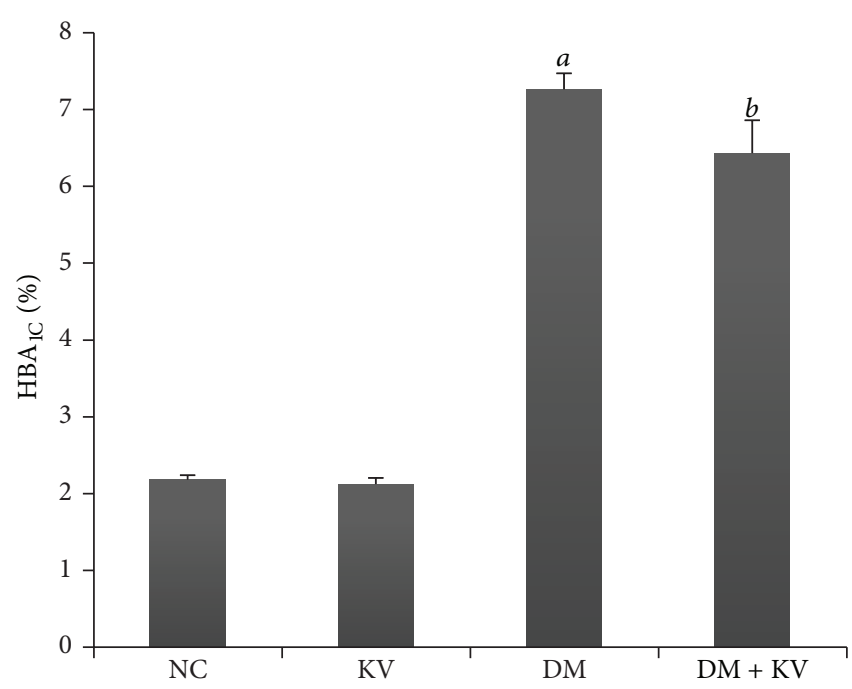

(b)

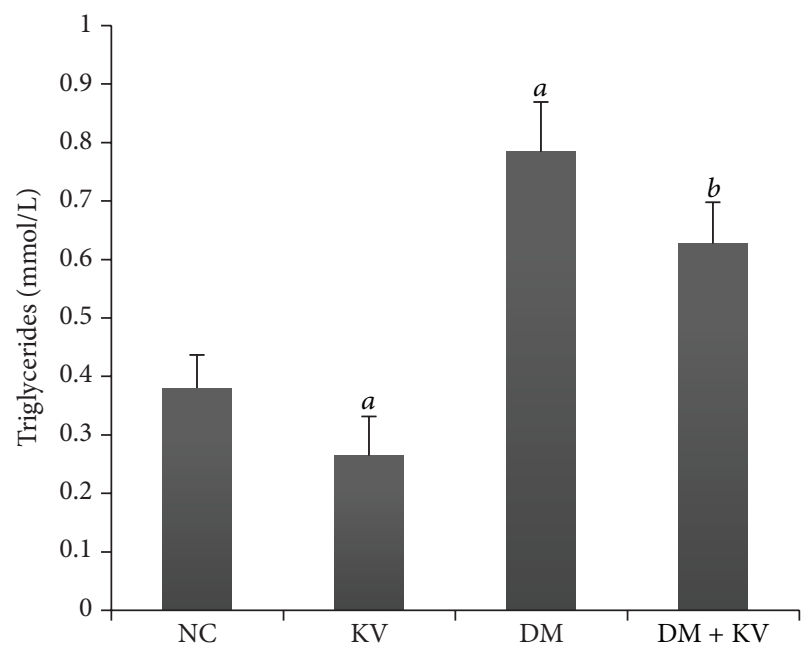

(d)

FIGURE 1: Effects of kolaviron on blood glucose, glycated haemoglobin, and levels of lipid profiles (total cholesterol and triglyceride concentrations). ${ }^{a}$ Values differ significantly from normal control $(P<0.05)$. ${ }^{b}$ Values differ significantly from diabetic control $(P<0.05)$. NC: normal control, KV: normal control treated with kolaviron, DM: untreated diabetic rats, DM + KV: diabetic rats treated with kolaviron.

and kolaviron normalized serum levels of these inflammatory markers in diabetic rats. No significant difference was observed in the serum levels of MCP-1, VEGF, and IL- $1 \beta$ in normal rats treated with kolaviron compared to untreated control group.

\section{Discussion}

The glucose lowering effect of kolaviron was observed in our study [31]. Glycated hemoglobin $\left(\mathrm{HBA}_{1 \mathrm{C}}\right)$, expressed as a percentage of total blood hemoglobin concentration, is an effective index for the screening of glycemic control over time. Higher level of $\mathrm{HBA}_{1 \mathrm{C}}$ is observed in diabetes due to reaction of excess blood glucose with hemoglobin. Evidence of glycemic regulation by kolaviron is the significant reduction of blood glucose and glycated hemoglobin levels in kolaviron supplemented diabetic rats.
Increased glucose concentration results in oxidative stress. Erythrocytes are vulnerable to oxidative stress due to high concentration of polyunsaturated fatty acids, ferrous ions, and molecular oxygen [32]. Persistent hyperglycemia and increased oxidative stress are major players in the development of secondary diabetic complications such as nephrotoxicity [33] and hepatic injury [34, 35]. Cells maintain a variety of defenses against reactive oxygen species toxicity and oxidative stress. Among these are an array of antioxidant enzymes including superoxide dismutase (SOD), catalase (CAT), and glutathione peroxidase (GPX). Superoxide dismutase (SOD) scavenges superoxide radical by accelerating its conversion to hydrogen peroxide $\left(\mathrm{H}_{2} \mathrm{O}_{2}\right)$ while glutathione peroxidase (GPX) detoxifies $\mathrm{H}_{2} \mathrm{O}_{2}$ and lipid peroxides $[36,37]$. CAT acts in the decomposition of hydrogen peroxide $\left(\mathrm{H}_{2} \mathrm{O}_{2}\right)$ to water and oxygen. Hyperglycemia can interfere with the antioxidant defense network 
TABLE 1: Effects of kolaviron on erythrocyte enzymatic activities and plasma lipid peroxidation in diabetic and normoglycemic rats.

\begin{tabular}{lcccc}
\hline & CAT & SOD & GPX & MDA \\
\hline NC & $0.34 \pm 0.06$ & $0.029 \pm 0.008$ & $32.13 \pm 4.60$ & $1.60 \pm 0.20$ \\
KV & $0.28 \pm 0.12$ & $0.026 \pm 0.008$ & $35.29 \pm 4.14$ & $1.5 \pm 0.21$ \\
DM & $0.37 \pm 0.07$ & $0.018 \pm 0.004^{\mathrm{a}}$ & $48.30 \pm 8.21^{\mathrm{a}}$ & $2.01 \pm 0.3^{\mathrm{a}}$ \\
DM + KV & $0.36 \pm 0.11$ & $0.022 \pm 0.006$ & $30.60 \pm 8.43^{\mathrm{b}}$ & $1.61 \pm 0.31^{\mathrm{b}}$ \\
\hline
\end{tabular}

Table 1: Illustrates the effect of kolaviron on erythrocyte enzymatic activities and plasma lipid peroxidation in diabetic and normoglycemic rats. CAT: catalase, $\mu \mathrm{mol} \mathrm{H}_{2} \mathrm{O}_{2}$ consumed/min/mg protein, SOD: superoxide dismutase, U/ $\mu \mathrm{g}$ protein, GPX: glutathione peroxidase, $\mu$ mol NADPH oxidized/min $/ \mu \mathrm{g}$ protein, MDA: malondialdehyde, $(\mu \mathrm{mol} \mathrm{MDA} / \mathrm{L})$. Data are presented as mean \pm S.D. ${ }^{\mathrm{a}}$ Values differ significantly from normal control $(P<0.05)$. ${ }^{\mathrm{b}}$ Values differ significantly from diabetic control $(P<0.05)$. NC: normal control; KV: normal control treated with kolaviron; DM: untreated diabetic rats; DM + KV: diabetic rats treated with kolaviron.

TABLE 2: Effects of kolaviron supplementation on plasma antioxidant status in diabetic rats.

\begin{tabular}{|c|c|c|c|}
\hline & ORAC $(\mu \mathrm{mol} \mathrm{TE} / \mathrm{L})$ & FRAP $(\mu \mathrm{mol}$ AAE/L $)$ & Total polyphenol (mg GAE/L) \\
\hline $\mathrm{NC}$ & $11.72 \pm 1.27$ & $85.68 \pm 9.45$ & $48.39 \pm 4.5$ \\
\hline KV & $13.57 \pm 1.54$ & $82.87 \pm 9.26$ & $47.84 \pm 3.3$ \\
\hline $\mathrm{DM}$ & $11.20 \pm 1.0$ & $80.60 \pm 8.56$ & $43.73 \pm 5$ \\
\hline $\mathrm{DM}+\mathrm{KV}$ & $11.03 \pm 1.71$ & $92.98 \pm 15.95$ & $46.45 \pm 6.15$ \\
\hline
\end{tabular}

Data as shown in Table 2 are presented as mean \pm S.D. NC: normal control; KV: normal control treated with kolaviron; DM: untreated diabetic rats; DM + KV: diabetic rats treated with kolaviron. AAE: ascorbic acid equivalent; TE: trolox equivalent; FRAP: ferric-reducing ability of the plasma; ORAC: oxygen radical absorbance capacity.

and the alteration in the activity of antioxidant enzymes is a common occurrence in diabetes. However divergent results have been reported regarding the activities of antioxidants enzymes in diabetics.

Alteration in antioxidant defense in the diabetic rats was evidenced by a significant reduction in SOD activity in the erythrocyte of diabetic rats. The decrease in SOD activity in the hyperglycemic rats could be due to oxidative stressinduced inactivation. Increased $\mathrm{H}_{2} \mathrm{O}_{2}$ concentration, for example, is known to inactivate SOD [38]. Glycosylation of SOD and/or loss of $\mathrm{Cu}^{2+}$, a cofactor required for SOD activity, can also reduce SOD activity [39]. We observed that supplementation of kolaviron to diabetic rat increased SOD activity to near normal level.

The observed increase in erythrocyte GPX activity in the unsupplemented diabetic rats is an indication of increased $\mathrm{H}_{2} \mathrm{O}_{2}$ concentration. Increased GPX activity might be due to an increase generation of $\mathrm{H}_{2} \mathrm{O}_{2}$ and a compensatory response to erythrocyte membrane oxidative damage. The reduction of oxidative stress in diabetic rats by kolaviron is evidenced by the suppression of GPX activity. Some studies have reported an increase in erythrocyte catalase activity in diabetic rats [40] while others have shown a decrease [41] in erythrocyte CAT activity. However, similar results to ours were found by Bandeira and colleagues [42] who observed no significant difference in erythrocytes CAT activity. The erythrocyte protective effects of kolaviron against free radical attack may be due to its direct free radical scavenging capacity and improvement in antioxidant defense.

The release of free radicals during oxidative stress causes serious damage to the biological system by abstracting electrons from macromolecules causing instability and disintegration. Antioxidants, in general, are compounds and reactions which dispose, scavenge, and suppress the formation of ROS [43]. The ability of kolaviron to protect against free radical induced damage and lipid peroxidation process is also evidenced by the significant decrease in the levels of malondialdehyde (MDA) - a product of lipid peroxidation in the plasma of diabetic rats following treatment with kolaviron. Kolaviron contains Garcinia biflavonoids. Flavonoids are a diverse group of polyphenols (phenyl benzopyrans) which function as phytochemicals and are well known for their multidirectional biological activities [44]. Structure-activity relationships have been shown to play a major role in the antioxidant effects of flavonoids. Structural features which confer antioxidant and free radical scavenging activity on kolaviron include its multiple aromatic hydroxyl groups [45, 46].

The ORAC and FRAP assays are two antioxidant capacity assays commonly used to assess the total antioxidant capacity of biological samples [24, 25]. The ORAC assay relies on free radical damage to a fluorescent probe, most commonly fluorescein, caused by an oxidizing reagent resulting in a loss of fluorescent intensity over time. The inhibition of oxidative damage to the fluorescent probe can be correlated with the antioxidant capacity of the compound. Also, the FRAP assay measures the ability of a sample to reduce $\mathrm{Fe}^{3+}$ to $\mathrm{Fe}^{2+}$ and reflects the plasma levels of ascorbic acid, uric acid, and $\alpha$-tocopherol [24] although it does not measure the $\mathrm{SH}$ group-containing antioxidants. The antioxidant activity of a compound against a free radical does not necessarily match its reducing ability. No significant difference was observed in plasma antioxidant capacity assessed by FRAP and ORAC assay between all the groups in our study.

Lipids are main sources of peroxidation products and elevation in lipid levels in diabetes mellitus plays an important role in the development of atherosclerosis and represents an increased risk factor for coronary heart diseases [47]. The 
TABLE 3: Effects of kolaviron treatment on levels of inflammatory proteins and growth factor.

\begin{tabular}{lccc}
\hline & IL-1 $\beta(\mathrm{pg} / \mathrm{mL})$ & MCP-1 $(\mathrm{pg} / \mathrm{mL})$ & VEGF $(\mathrm{pg} / \mathrm{mL})$ \\
\hline NC & $28.37 \pm 10.54$ & $226.62 \pm 75.71$ & $10.45 \pm 1.4$ \\
KV & $20.48 \pm 5.13$ & $231.26 \pm 78.31$ & $9.49 \pm 2.5$ \\
DM & $43.32 \pm 8.65^{\mathrm{a}}$ & $542.77 \pm 67.27^{\mathrm{a}}$ & $15.41 \pm 1.96^{\mathrm{a}}$ \\
$\mathrm{DM}+\mathrm{KV}$ & $16.43 \pm 7.74^{\mathrm{b}}$ & $266.61 \pm 81.36^{\mathrm{b}}$ & $12.44 \pm 1.73^{\mathrm{b}}$ \\
\hline
\end{tabular}

Table 3 shows effects of kolaviron on interleukin (IL)-1 $\beta$, monocyte chemotactic protein (MCP-1), and vascular endothelial growth factor (VEGF). Data are presented as mean \pm S.D. ${ }^{a}$ Values differ significantly from normal control $(P<0.05) .{ }^{\mathrm{b}}$ Values differ significantly from diabetic control $(P<0.05)$. NC: normal control; KV: normal control treated with kolaviron; DM: untreated diabetic rats; DM + KV: diabetic rats treated with kolaviron.

hyperlipidemia observed in the untreated diabetic rats in the present study could indicate an increase in the mobilization of free fatty acids from the peripheral fat depots. This could result from the uninhibited actions of lipolytic enzyme lipase caused by insulin deficiency characteristic of the diabetic state. We observed in the present study that kolaviron significantly lowered total cholesterol and triglyceride levels in the serum of normal and diabetic rats.

Hyperglycaemia can result in elevated levels of circulating inflammatory mediators [48]. Increase in oxidative stress can increase the production of inflammatory proteins and vice versa. Interaction between oxidative stress and inflammatory signals plays a major role in disease progression and tissue damage in diabetes $[49,50]$. Elevated levels of VEGF, IL-1, and MCP-1 have been implicated in diabeticrelated complications [51-53]. VEGF is an angiogenic factor with potent vascular permeability and angiogenic effects, plays a central role in vasculogenesis and neoangiogenesis by promoting the survival, migration, and proliferation of endothelial cells, and regulates glomerular permeability [54]. Our findings agree with previous report of elevated serum levels of VEGF in diabetes [55]. Despite its protective role in nondiabetic renal disease, overexpression of VEGF is implicated in diabetic renal disease by increasing the permeability of vascular endothelium, endothelial cell proliferation and migration, and activation of matrix-degrading and plays a major pathophysiological role in diabetic nephropathy. Factors that modulate expression of VEGF and its receptors are high glucose, AGEs, endothelin 1, angiotensin II, and TGF- $\beta$ [56]. In the present study, kolaviron normalized VEGF concentration in the serum of diabetic rats. The role of IL- $1 \beta$ has been well reported in diabetes. IL- $1 \beta$ increases the expression of chemotactic factors and adhesion molecules, enhances vascular endothelial permeability, and stimulates the proliferation of mesangial cells and matrix synthesis [49]. Kolaviron significantly reduced IL- $1 \beta$ mediated inflammation in the serum of diabetic rats in comparison to untreated diabetic control rats.

Increased production of monocyte chemoattractant protein (MCP-1) can occur after induction of oxidative stress or growth factors in a number of cells such as monocytes, smooth muscle cells, and endothelial cells [57]. MCP-1 mediated macrophage accumulation plays a role in the formation of vascular inflammation, atherosclerotic lesion, and kidney damage [58-60]. The protective effect of kolaviron in our study is evidenced by the restoration of MCP-1 to near normal levels in diabetic rats.
Overall, our study showed the protective effects of kolaviron in the blood of diabetic rats through improvement in glucose level, endogenous antioxidant defense, and inflammation. Increased activation of major pathways such as advanced glycation end products (AGEs), protein kinase C $(\mathrm{PKC})$, and aldose reductase due to a hyperglycemic state is thought to amplify the production of free radicals and inflammatory biomarkers in diabetes, hence mediating the damaging effect. The ability of kolaviron to interfere with one or more of these pathways in the present study could possibly explain the observed beneficial effects of kolaviron in the blood of diabetic rats.

\section{Conclusion}

The result of this study indicates that the mechanism of antidiabetic effects of kolaviron may be related to its intrinsic antioxidative and anti-inflammatory properties and suggests that kolaviron may be beneficial in reducing the risk of vascular complications in diabetes.

\section{Conflict of Interests}

The authors declare that there is no conflict of interests regarding the publication of this paper.

\section{Acknowledgments}

This study was supported by the University Research Fund (URF) of the Cape Peninsula University of Technology and the National Research Foundation, South Africa (NRF), granted to Professor Oluwafemi O. Oguntibeju.

\section{References}

[1] L. L. Baggio and D. J. Drucker, "Biology of Incretins: GLP-1 and GIP,” Gastroenterology, vol. 132, no. 6, pp. 2131-2157, 2007.

[2] M. Brownlee, "Biochemistry and molecular cell biology of diabetic complications," Nature, vol. 414, no. 6865, pp. 813-820, 2001.

[3] P. Rösen, P. P. Nawroth, G. King, W. Möller, H.-J. Tritschler, and L. Packer, "The role of oxidative stress in the onset and progression of diabetes and its complications: a summary of a congress series sponsored by UNESCO-MCBN, the American diabetes association and the German diabetes society," Diabetes/Metabolism Research and Reviews, vol. 17, no. 3, pp. 189212, 2001. 
[4] J. L. Evans, I. D. Goldfine, B. A. Maddux, and G. M. Grodsky, "Oxidative stress and stress-activated signaling pathways: a unifying hypothesis of type 2 diabetes," Endocrine Reviews, vol. 23, no. 5, pp. 599-622, 2002.

[5] T. Bahorun, M. Soobrattee, V. Luximon-Ramma, and O. Aruoma, "Free radicals and antioxidants in cardiovascular health and disease," Internet Journal of Medical Update, vol. 1, no. 2, pp. 25-41, 2006.

[6] B. Halliwell, "Biochemistry of oxidative stress," Biochemical Society Transactions, vol. 35, part 5, pp. 1147-1150, 2007.

[7] K. B. Pandey and S. I. Rizvi, "Biomarkers of oxidative stress in red blood cells," Biomedical Papers, vol. 155, no. 2, pp. 131-136, 2011.

[8] S. Comazzi, V. Spagnolo, and U. Bonfanti, "Erythrocyte changes in canine diabetes mellitus: in vitro effects of hyperglycaemia and ketoacidosis," Comparative Clinical Pathology, vol. 12, no. 4, pp. 199-205, 2004.

[9] M. Dallak and I. Bin-Jaliah, "Antioxidant activity of Citrulluscolocynthis pulp extract in the RBC's of alloxan-induced diabetic rats," Pakistan Journal of Physiology, vol. 6, no. 1, pp. 112-122, 2010.

[10] K. Kolanjiappan, S. Manoharan, and M. Kayalvizhi, "Measurement of erythrocyte lipids, lipid peroxidation, antioxidants and osmotic fragility in cervical cancer patients," Clinica Chimica Acta, vol. 326, no. 1-2, pp. 143-149, 2002.

[11] S. Kiritoshi, T. Nishikawa, K. Sonoda et al., "Reactive oxygen species from mitochondria induce cyclooxygenase-2 gene expression in human mesangial cells: potential role in diabetic nephropathy," Diabetes, vol. 52, no. 10, pp. 2570-2577, 2003.

[12] J. W. Baynes, "Chemical modification of proteins by lipids in diabetes," Clinical Chemistry and Laboratory Medicine, vol. 41, no. 9, pp. 1159-1165, 2003.

[13] S. P. Wolff, "Diabetes mellitus and free radicals. Free radicals, transition metals and oxidative stress in the aetiology of diabetes mellitus and complications," British Medical Bulletin, vol. 49, no. 3, pp. 642-652, 1993.

[14] A. Griesmacher, M. Kindhauser, S. E. Andert et al., "Enhanced serum levels of thiobarbituric-acid-reactive substances in diabetes mellitus," American Journal of Medicine, vol. 98, no. 5, pp. 469-475, 1995.

[15] J. F. Navarro-González, C. Mora-Fernández, M. M. de Fuentes, and J. García-Pérez, "Inflammatory molecules and pathways in the pathogenesis of diabetic nephropathy," Nature Reviews Nephrology, vol. 7, no. 6, pp. 327-340, 2011.

[16] R.-E. W. Kavey, V. Allada, S. R. Daniels et al., "Cardiovascular risk reduction in high-risk pediatric patients: a scientific statement from the American Heart Association expert panel on population and prevention science; the councils on cardiovascular disease in the young, epidemiology and prevention, nutrition, physical activity and metabolism, high blood pressure research, cardiovascular nursing, and the kidney in heart disease; and the Interdisciplinary Working Group on Quality of Care and Outcomes Research: endorsed by the American Academy of Paediatrics," Circulation, vol. 114, no. 24, pp. 2710 2738, 2006.

[17] K. Terashima, Y. Takaya, and M. Niwa, "Powerful antioxidative agents based on garcinoic acid from Garcinia kola," Bioorganic and Medicinal Chemistry, vol. 10, no. 5, pp. 1619-1625, 2002.

[18] S. B. Olaleye, E. O. Farombi, E. A. Adewoye, B. V. Owoyele, S. A. Onasanwo, and R. A. Elegbe, "Analgesic and anti-inflammatory effects of kolaviron (a Garcinia kola seed extract)," African Journal of Biomedical Research, vol. 3, pp. 171-174, 2000.
[19] E. O. Farombi, B. F. Adepoju, O. E. Ola-Davies, and G. O. Emerole, "Chemoprevention of aflatoxin B1-induced genotoxicity and hepatic oxidative damage in rats by kolaviron, a natural biflavonoid of Garcinia kola seeds," European Journal of Cancer Prevention, vol. 14, no. 3, pp. 207-214, 2005.

[20] E. O. Farombi, S. Shrotriya, and Y.-J. Surh, "Kolaviron inhibits dimethyl nitrosamine-induced liver injury by suppressing COX- 2 and iNOS expression via NF- $\kappa$ B and AP-1," Life Sciences, vol. 84, no. 5-6, pp. 149-155, 2009.

[21] I. A. Adedara and E. O. Farombi, "Chemoprotection of ethylene glycol monoethyl ether-induced reproductive toxicity in male rats by kolaviron, isolated biflavonoid from Garcinia kola seed," Human and Experimental Toxicology, vol. 31, pp. 506-517, 2012.

[22] E. O. Farombi, I. A. Adedara, B. O. Ajayi, O. R. Ayepola, and E. E. Egbeme, "Kolaviron, a natural antioxidant and antiinflammatory phytochemical prevents dextran sulphate sodium-induced colitis in rats," Basic and Clinical Pharmacology and Toxicology, vol. 113, no. 1, pp. 49-55, 2013.

[23] M. M. Iwu, O. A. Igboko, C. O. Okunji, and M. S. Tempesta, "Antidiabetic and aldose reductase activities of biflavanones of Garcinia kola," Journal of Pharmacy and Pharmacology, vol. 42, no. 4, pp. 290-292, 1990.

[24] I. F. F. Benzie and J. J. Strain, "The ferric reducing ability of plasma (FRAP) as a measure of "antioxidant power": the FRAP assay," Analytical Biochemistry, vol. 239, no. 1, pp. 70-76, 1996.

[25] B. Ou, M. Hampsch-Woodill, and R. L. Prior, "Development and validation of an improved oxygen radical absorbance capacity assay using fluorescein as the fluorescent probe," Journal of Agricultural and Food Chemistry, vol. 49, no. 10, pp. 4619-4626, 2001.

[26] V. L. Singleton, R. Orthofer, and R. M. Lamuela-Raventós, "Analysis of total phenols and other oxidation substrates and antioxidants by means of folin-ciocalteu reagent," Methods in Enzymology, vol. 299, pp. 152-178, 1999.

[27] H. Aebi, "Catalase in vitro," Methods in Enzymology, vol. 105, pp. 121-126, 1984.

[28] N. Crosti, T. Servidei, J. Bajer, and A. Serra, "Modification of the 6-hydroxydopamine technique for the correct determination of superoxide dismutase," Journal of Clinical Chemistry and Clinical Biochemistry, vol. 25, no. 4, pp. 265-266, 1987.

[29] L. M. Ellerby and D. E. Bredesen, "Measurement of cellular oxidation, reactive oxygen species, and antioxidant enzymes during apoptosis," Methods in Enzymology, vol. 322, pp. 413-421, 2000.

[30] G. A. Khoschsorur, B. M. Winklhofer-Roob, H. Rabl, T. Auer, Z. Peng, and R. J. Schaur, "Evaluation of a sensitive HPLC method for the determination of malondialdehyde, and application of the method to different biological materials," Chromatographia, vol. 52, no. 3-4, pp. 181-184, 2000.

[31] O. R. Ayepola, N. N. Chegou, N. L. Brooks, and O. O. Oguntibeju, "Kolaviron, a Garcinia biflavonoid complex ameliorates hyperglycemia-mediated hepatic injury in rats via suppression of inflammatory responses," BMC Complementary and Alternative Medicine, vol. 13, article 363, 2013.

[32] P. Yadav, S. Sarkar, and D. Bhatnagar, "Lipid peroxidation and antioxidant enzymes in erythrocytes and tissues in aged diabetic rats," Indian Journal of Experimental Biology, vol. 35, no. 4, pp. 389-392, 1997.

[33] C. Huang, Y. Kim, M. L. A. Caramori et al., "Cellular basis of diabetic nephropathy: II. The transforming growth factor- $\beta$ system and diabetic nephropathy lesions in type 1 diabetes," Diabetes, vol. 51, no. 12, pp. 3577-3581, 2002. 
[34] J. Y. Kim, S. H. Lee, E. H. Song et al., "A critical role of STAT1 in streptozotocin-induced diabetic liver injury in mice: controlled by ATF3," Cellular Signalling, vol. 21, no. 12, pp. 1758-1767, 2009.

[35] S. A. Harrison, "Liver disease in patients with diabetes mellitus," Journal of Clinical Gastroenterology, vol. 40, no. 1, pp. 68-76, 2006.

[36] I. N. Zelko, T. J. Mariani, and R. J. Folz, "Superoxide dismutase multigene family: a comparison of the CuZn-SOD (SOD1), MnSOD (SOD2), and EC-SOD (SOD3) gene structures, evolution, and expression," Free Radical Biology and Medicine, vol. 33, no. 3, pp. 337-349, 2002.

[37] S. Jurkovič, J. Osredkar, and J. Marc, "Molecular impact of glutathione peroxidases in antioxidant processes," Biochemia Medica, vol. 18, no. 2, pp. 162-174, 2008.

[38] A. Seven, S. Güzel, O. Seymen et al., "Effects of vitamin E supplementation on oxidative stress in streptozotocin induced diabetic rats: investigation of liver and plasma," Yonsei Medical Journal, vol. 45, no. 4, pp. 703-710, 2004.

[39] K. Hamden, S. Carreau, K. Jamoussi et al., " $1 \alpha$,25 dihydroxyvitamin D3: therapeutic and preventive effects against oxidative stress, hepatic, pancreatic and renal injury in alloxan-induced diabetes in rats," Journal of Nutritional Science and Vitaminology, vol. 55, no. 3, pp. 215-222, 2009.

[40] A. Likidlilid, N. Patchanans, T. Peerapatdit, and C. Sriratanasathavorn, "Lipid peroxidation and antioxidant enzyme activities in erythrocytes of type 2 diabetic patients," Journal of the Medical Association of Thailand, vol. 93, no. 6, pp. 682-693, 2010.

[41] K. Z. Kędziora-Kornatowska, M. Luciak, J. Blaszczyk, and W. Pawlak, "Lipid peroxidation and activities of antioxidant enzymes in erythrocytes of patients with non-insulin dependent diabetes with or without diabetic nephropathy," Nephrology Dialysis Transplantation, vol. 13, no. 11, pp. 2829-2832, 1998.

[42] M. Bandeira, S. Guedes, L. J. da Fonseca et al., "Characterization of blood oxidative stress in type 2 diabetes mellitus patients: increase in lipid peroxidation and SOD activity," Oxidative Medicine and Cellular Longevity, vol. 2012, Article ID 819310, 13 pages, 2012.

[43] M. Valko, D. Leibfritz, J. Moncol, M. T. D. Cronin, M. Mazur, and J. Telser, "Free radicals and antioxidants in normal physiological functions and human disease," International Journal of Biochemistry and Cell Biology, vol. 39, no. 1, pp. 44-84, 2007.

[44] E. Corradini, P. Foglia, P. Giansanti, R. Gubbiotti, R. Samperi, and A. Laganà, "Flavonoids: chemical properties and analytical methodologies of identification and quantitation in foods and plants," Natural Product Research, vol. 25, no. 5, pp. 469-495, 2011.

[45] E. O. Farombi and I. A. Nwaokeafor, "Anti-oxidant mechanisms of kolaviron: studies on serum lipoprotein oxidation, metal chelation and oxidative membrane damage in rats," Clinical and Experimental Pharmacology and Physiology, vol. 32, no. 8, pp. 667-674, 2005.

[46] E. O. Farombi, S. O. Abarikwu, I. A. Adedara, and M. O. Oyeyemi, "Curcumin and kolaviron ameliorate di-n-butylphthalate-induced testicular damage in rats," Basic and Clinical Pharmacology and Toxicology, vol. 100, no. 1, pp. 43-48, 2007.

[47] J. Betteridge, "Lipid disorders in diabetes mellitus," in Text Book of Diabetes, J. C. Pickup and G. Williams, Eds., pp. 1-35, Blackwell Science, London, UK, 2nd edition, 1997.

[48] K. Esposito, F. Nappo, R. Marfella et al., "Inflammatory cytokine concentrations are acutely increased by hyperglycemia in humans: role of oxidative stress," Circulation, vol. 106, no. 16, pp. 2067-2072, 2002.

[49] A. A. Elmarakby and J. C. Sullivan, "Relationship between oxidative stress and inflammatory cytokines in diabetic nephropathy," Cardiovascular Therapeutics, vol. 30, no. 1, pp. 49-59, 2012.

[50] G. P. A. Vadla and E. Vellaichamy, "Beneficial effects of aminoguanidine against streptozotocin-induced pathological changes in diabetic mice kidney," Biomedicine \& Preventive Nutrition, vol. 3, no. 3, pp. 221-226, 2012.

[51] F. Santilli, A. Spagnoli, A. Mohn et al., "Increased vascular endothelial growth factor serum concentrations may help to identify patients with onset of type 1 diabetes during childhood at risk for developing persistent microalbuminuria," Journal of Clinical Endocrinology and Metabolism, vol. 86, no. 8, pp. 38713876, 2001.

[52] G. H. Tesch, "MCP-1/CCL2: a new diagnostic marker and therapeutic target for progressive renal injury in diabetic nephropathy," American Journal of Physiology-Renal Physiology, vol. 294, no. 4, pp. F697-F701, 2008.

[53] P. Palsamy and S. Subramanian, "Resveratrol protects diabetic kidney by attenuating hyperglycemia-mediated oxidative stress and renal inflammatory cytokines via Nrf2-Keap1 signaling," Biochimica et Biophysica Acta, vol. 1812, no. 7, pp. 719-731, 2011.

[54] H. Takahashi and M. Shibuya, "The vascular endothelial growth factor (VEGF)/VEGF receptor system and its role under physiological and pathological conditions," Clinical Science, vol. 109, no. 3, pp. 227-241, 2005.

[55] F. Chiarelli, A. Spagnoli, F. Basciani et al., "Vascular endothelial growth factor (VEGF) in children, adolescents and young adults with type 1 diabetes mellitus: relation to glycaemic control and microvascular complications," Diabetic Medicine, vol. 17, no. 9, pp. 650-656, 2000.

[56] S. Chen and F. N. Ziyadeh, "Vascular endothelial growth factor and diabetic nephropathy," Current Diabetes Reports, vol. 8, no. 6, pp. 470-476, 2008.

[57] L. Xing and D. G. Remick, "Promoter elements responsible for antioxidant regulation of MCP-1 gene expression," Antioxidants and Redox Signaling, vol. 9, no. 11, pp. 1979-1989, 2007.

[58] D. J. Lefer and D. N. Granger, "Monocyte rolling in early atherogenesis vital role in lesion development," Circulation Research, vol. 84, no. 11, pp. 1353-1355, 1999.

[59] K. Egashira, "Molecular mechanisms mediating inflammation in vascular disease: special reference to monocyte chemoattractant protein-1," Hypertension, vol. 41, no. 3, pp. 834-841, 2003.

[60] F. Y. Chow, D. J. Nikolic-Paterson, E. Ozols, R. C. Atkins, B. J. Rollin, and G. H. Tesch, "Monocyte chemoattractant protein1 promotes the development of diabetic renal injury in streptozotocin-treated mice," Kidney International, vol. 69, no. 1, pp. 73-80, 2006. 

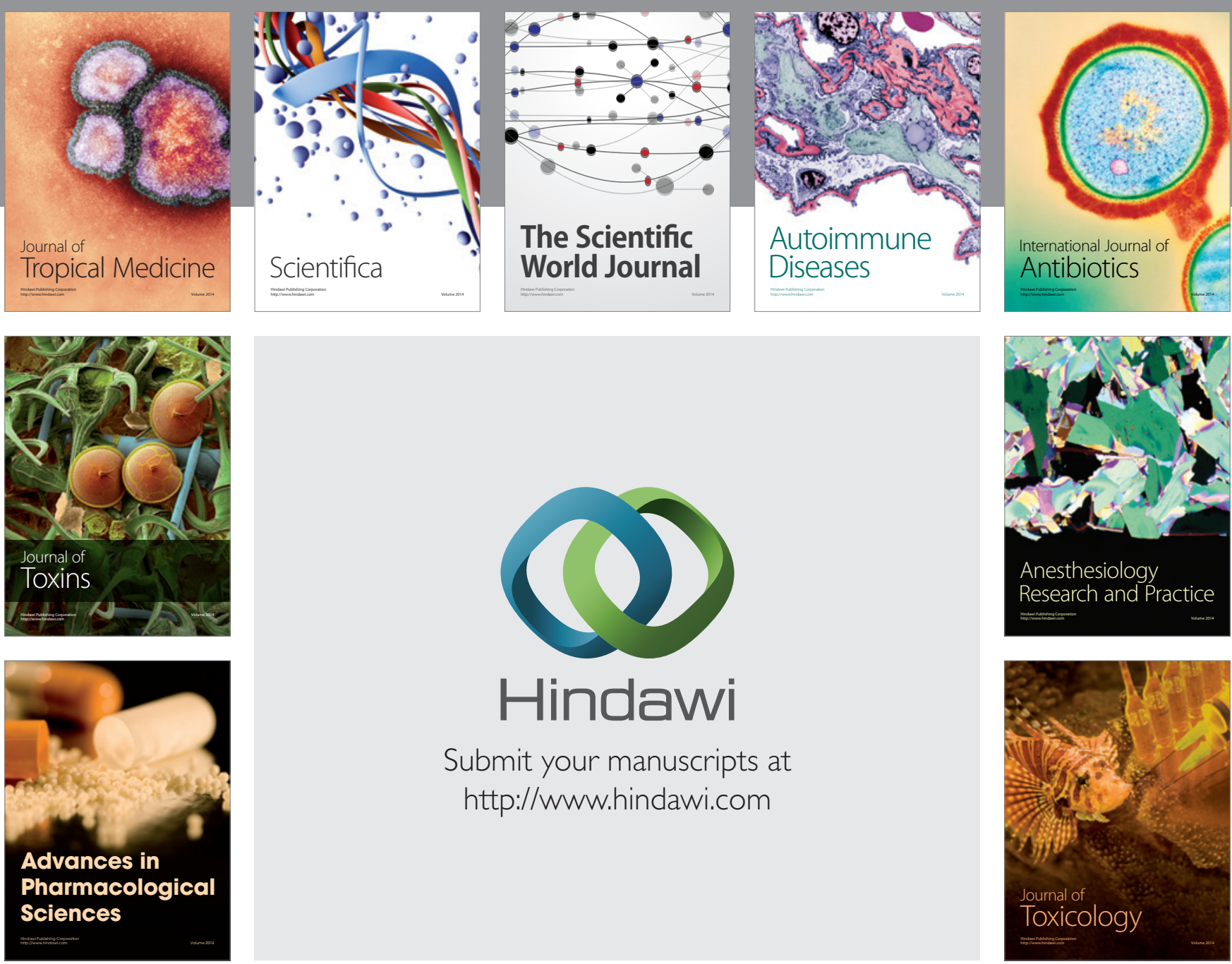

\section{Hindawi}

Submit your manuscripts at

http://www.hindawi.com
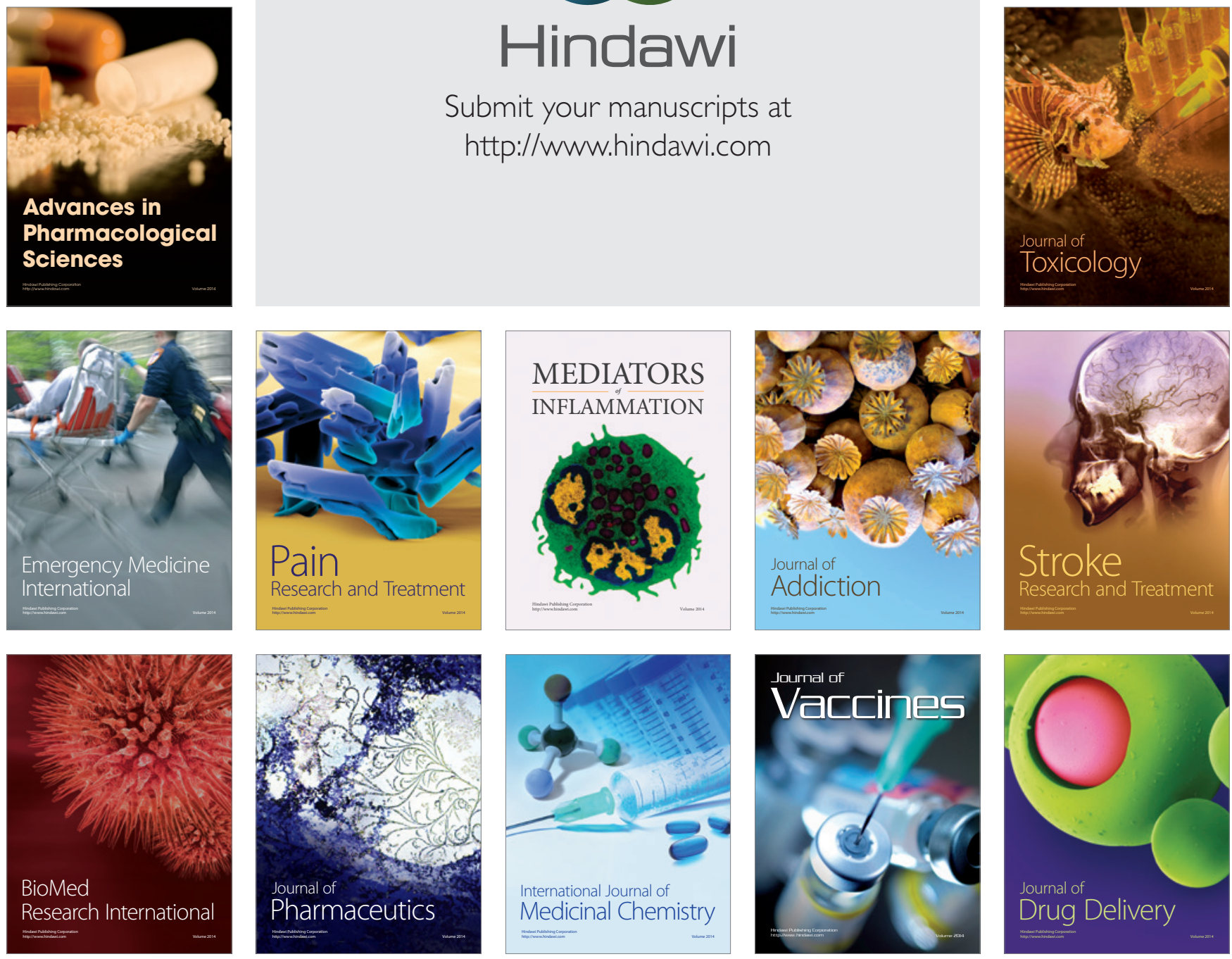